\title{
Parity Violation in the NN System
}

\author{
R.P. Springer ${ }^{1, a}$, D.R. Phillips ${ }^{2}$, and M.J. Schindler ${ }^{3}$ \\ 1 Duke University, Department of Physics, Durham, NC 27708 \\ 2 Ohio University, Department of Physics and Astronomy, Athens, OH 45701 \\ 3 George Washington University, Department of Physics, Washington, DC, 20052
}

\begin{abstract}
We briefly review the apparent experimental discrepancies that sustain interest in the field of lowenergy few-nucleon parity violation. We argue that it is not possible to determine whether present experimental measurements are consistent unless each is understood in terms of a complete EFT with consistent power counting. Towards this end, we present the EFT that describes very low energy parity violating observables associated with two-nucleon scattering and photon-deuteron interactions.
\end{abstract}

\section{Introduction}

In this talk we will use an effective field theory to explore parity violation (PV) in the two-nucleon system. While few measurements are presently available for comparison to theory, the experimental community recognizes the importance of the system and several new results should be available within the next five years. Including three and more nucleon PV processes will ultimately be necessary to complete our understanding. But those calculations depend upon the systematic treatment of the two-nucleon system, and so we begin there.

The standard model of weak interactions contains PV expressed in terms of quarks, weak bosons, etc. But these are not the degrees of freedom that are appropriate for understanding $\mathrm{PV}$ in few nucleon systems at low energy. At low enough energies, the effective degrees of freedom are the nucleons and photons; all other excitations, including not only quarks, gluons, and weak bosons, but also pions are subsumed into contact interactions. Because QCD cannot be matched onto this "pionless EFT," $\operatorname{EFT}(\not)$, we are left with a number of unknown parameters that must be set by experimental or lattice data. With enough data, we can determine whether the EFT, with its attendant power counting, is consistent with reality. The standard model of weak interactions comes from

$$
\begin{array}{r}
\mathcal{L}_{\mathrm{SM}}=\frac{g}{2 \sqrt{2}}(\bar{u} \bar{c} \bar{t}) \gamma^{\mu}\left(1-\gamma_{5}\right) V\left(\begin{array}{l}
d \\
s \\
b
\end{array}\right) W_{\mu}^{+}+\text {h.c. } \\
+[\text { terms involving } Z]+\cdots
\end{array}
$$

(where $V$ is the CKM matrix), from which the necessary weak interaction Hamiltonian is found (see, e.g., Ref. [1])

$$
\mathcal{H}=\frac{G_{F}}{\sqrt{2}}\left(\left[J_{(c c)}^{v}\right]^{\dagger} J_{(c c) v}+\left[J_{(n c)}^{v}\right]^{\dagger} J_{(n c) v}\right),
$$

\footnotetext{
${ }^{a}$ e-mail: rps@phy.duke.edu
}

where the charged current

$$
J_{(c c)}^{v} \sim \bar{u} \gamma^{v}\left(1-\gamma_{5}\right)\left[\cos \theta_{c} d+\sin \theta_{c} s\right]
$$

and the neutral current

$$
\begin{aligned}
J_{(n c)}^{v} & \sim \bar{u} \gamma^{v}\left(1-\gamma_{5}\right) u-\bar{d} \gamma^{v}\left(1-\gamma_{5}\right) d \\
& -\bar{s} \gamma^{v}\left(1-\gamma_{5}\right) s-4 \sin ^{2} \theta_{W} J_{E M}^{v}
\end{aligned}
$$

and $G_{F}$ is Fermi's weak constant. For nucleon interactions, we are interested in the portion of $\mathcal{H}$ that does not change strangeness. At the same time, the only way to extract this $\Delta S=0$ component from nucleon interactions without being overwhelmed by strong interactions is to look at PV quantities since the ratio of weak to strong interactions $\sim 10^{-7}$. The $\Delta S=0$ part of the interaction Hamiltonian can be decomposed into three pieces based upon the change in isospin: $\Delta I=2, \Delta I=1$, and $\Delta I=0$. Because of the smallness of the Cabbibo angle $\theta_{c}$, the $\Delta I=1$ portion is dominated by the neutral current.

From, for example, Fig. 5 in Ref. [2], it would appear that the available measurements of PV observables (seen in pp scattering, in p-He scattering, and in heavier systems where nearby parity states create enhancements) are inconsistent with the theoretical framework in which they are interpreted. However, that framework, known as the DDH model [3] does not necessarily coincide with the predictions of QCD. The DDH model is based upon a meson exchange picture, including vector mesons, which are either not dynamical (at low energies) or, where they are dynamical, are not included in a systematic way. Perhaps just as seriously, the nuclear and atomic corrections in the heavier systems may not be under control. To address the former issue, a consistent low-energy EFT with defendable power counting was presented in Ref. [4]. To address the latter issue may require large-scale computations that respect the appropriate EFT symmetries and power counting. 


\section{Lagrangian}

Crucially for the success of low-energy few nucleon PV calculations, the analogous strong-interaction problem is well understood. Restricted to the two-nucleon case, the parity conserving $\mathcal{L}$ is [5]

$$
\begin{aligned}
\mathcal{L}_{P C} & =N^{\dagger}\left(i D_{0}+\frac{\mathbf{D}^{2}}{2 M}\right) N+\frac{e}{2 M} N^{\dagger}\left(\kappa_{0}+\tau_{3} \kappa_{1}\right) \sigma \cdot \boldsymbol{B} N \\
& -C_{0}^{\left({ }^{(} S_{0}\right)}\left(N^{T} P_{a}^{\left(1 S_{0}\right)} N\right)^{\dagger}\left(N^{T} P_{a}^{\left(1 S_{0}\right)} N\right) \\
& -C_{0}^{\left({ }^{3} S_{1}\right)}\left(N^{T} P_{i}^{\left({ }^{3} S_{1}\right)} N\right)^{\dagger}\left(N^{T} P_{i}^{\left({ }^{3} S_{1}\right)} N\right)+\ldots
\end{aligned}
$$

where $P_{a}^{\left({ }^{(} S_{0}\right)}=\frac{1}{\sqrt{8}} \tau_{2} \tau_{a} \sigma_{2}$ and $P_{i}^{\left(3 S_{1}\right)}=\frac{1}{\sqrt{8}} \tau_{2} \sigma_{2} \sigma_{i}$ project onto the appropriate partial waves (the $\sigma_{i}$ and $\tau_{a}$ are SU(2) Pauli matrices in spin and isospin space, respectively), $D_{\mu} N=\partial_{\mu} N+i e \frac{1+\tau_{3}}{2} A_{\mu} N$, and $C_{0}^{\left({ }^{1} S_{0}\right)}$ and $C_{0}^{\left({ }^{3} S_{1}\right)}$ can be extracted from experiment. In the power divergence subtraction scheme $[6,7]$

$$
\begin{aligned}
& C_{0}^{\left(1 S_{0}\right)}=\frac{4 \pi}{M} \frac{1}{\frac{1}{a^{\left.1 S_{0}\right)}}-\mu}, \\
& C_{0}^{\left(3 S_{1}\right)}=\frac{4 \pi}{M} \frac{1}{\frac{1}{a^{\left.3 S_{1}\right)}}-\mu},
\end{aligned}
$$

where $a^{\left(1 S_{0}\right)}$ and $a^{\left({ }^{3} S_{1}\right)}$ are the scattering lengths in the singlet and triplet $S$-channel, respectively. This subtraction point dependence will also be seen in the parameters entering the weak portion of the two-nucleon $\operatorname{EFT}(\not)$.

The $S$-wave strong interaction chain in Fig. 1 must be

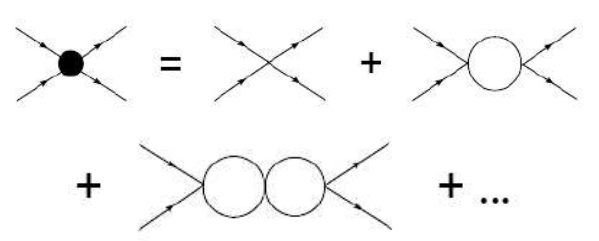

Fig. 1. Infinite bubble chain for nucleon-nucleon scattering under contact interactions that encode strong interactions.

summed to all orders because of the anomalously large scattering length in both the singlet and triplet channels: $a^{\left({ }^{1} S_{0}\right)} \sim-1 /(8 \mathrm{MeV}), a^{\left({ }^{3} S_{1}\right)} \sim 1 /(36 \mathrm{MeV})$. But while the momentum expansion fails, this sum can be performed to yield

$$
A=-\frac{4 \pi}{M} \frac{1}{1 / a+i p}+\cdots
$$

Electromagnetic effects have been successfully included and agree well with experiment (See, e.g., Ref. $[9,10]$ ). With $\operatorname{EFT}(\not)$ well established for both the strong and electromagnetic sectors, it remains to extend the treatment to include parity violation. The two-nucleon parity violating observables include
- $\vec{p} p$ analyzing power. Polarized protons are scattered off unpolarized protons. The asymmetry is the difference between the (differential) cross section when the protons are polarized along their direction of motion, $\sigma^{+}(\theta)$, versus when the protons are polarized antiparallel to their direction of motion, $\sigma^{-}(\theta)$ :

$$
A_{L}=\frac{\sigma^{+}(\theta)-\sigma^{-}(\theta)}{\sigma^{+}(\theta)+\sigma^{-}(\theta)}
$$

This has been measured at $13.6 \mathrm{MeV}$ by Eversheim et al. [11] to be $(-0.93 \pm 0.21) \times 10^{-7}$.

- $\vec{n} n$ analyzing power.

- $\vec{n} p$ analyzing power.

- $n+p \rightarrow d+\gamma$. (Or its time-reversed process.) The circular polarization of the photon after capture (or the polarized photon breakup of the deuteron at the same kinematic point) yields an asymmetry presently consistent with zero: $P_{\gamma}=\frac{\sigma^{+}-\sigma^{-}}{\sigma^{+}+\sigma^{-}}=(1.8 \pm 1.8) \times 10^{-7}[12,13]$. The existence of FEL programs such as that utilized by TUNL at Duke may provide a new measurement of the breakup reaction if future upgrades are approved.

- $\vec{n}+p \rightarrow d+\gamma$. The angular distribution of the photon emitted by polarized neutron capture is also consistent with zero, $A_{\gamma}=(0.6 \pm 2.1) \times 10^{-7}[14,15]$, where $\frac{1}{\Gamma} \frac{d \Gamma}{d \cos \theta}=1+A_{\gamma} \cos \theta$, but a new measurement is expected from NPDGamma at Oak Ridge [16].

Considering more nucleons in the process provides additional observables. Some of those involving polarized neutrons are being considered at the SNS. The technology exists to make such predictions in $\operatorname{an} \operatorname{EFT}(\not)$ framework, but that is beyond the scope of this talk. Ultimately they will be needed to fully determine the consistency of the PV $\operatorname{EFT}(\hbar)$ description.

A convenient categorization of the PV operators is obtained by using the partial wave basis; the same basis that was chosen to present the parity conserving Lagrangian in Eqn. (3). At leading order there are only five possibilities $[17,4,18]:{ }^{3} S_{1} \leftrightarrow{ }^{1} P_{1}$, which is isospin change $\Delta I=0$ and describes only $n p$ scattering (not $n n$ or $p p$ ); ${ }^{1} S_{0} \leftrightarrow{ }^{3} P_{0}$ with $\Delta I=0$ (nn, np, or pp interactions); ${ }^{1} S_{0} \leftrightarrow{ }^{3} P_{0}$ with $\Delta I=1$ (nn or pp only); ${ }^{1} S_{0} \leftrightarrow{ }^{3} P_{0}$ with $\Delta I=2$ (nn, $n p$, or $p p$ ); and ${ }^{3} S_{1} \leftrightarrow{ }^{3} P_{1}$, which is $\Delta I=1$ ( $n p$ only). $P-D$ wave transitions enter at higher order.

The concept of creating effective operators to build a field theory to include the above terms is analogous to Fermi's four-point operator. At low enough energies, the details of the weak interaction are not accessible. For example, in $\beta$ decay, the process is described by a parameter, $G_{F}$, multiplied by a contact interaction, shown in Fig. 2.

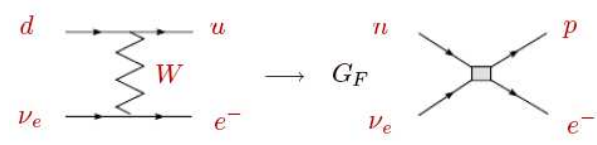

Fig. 2. Low energy beta decay mapped to EFT. 
For low energy PV, we again encode not only strong interactions of the quarks binding to form nucleons, but the weak interactions as a sum of contact operators, each multiplied by an unknown parameter, as in Fig. 3. Explicitly,

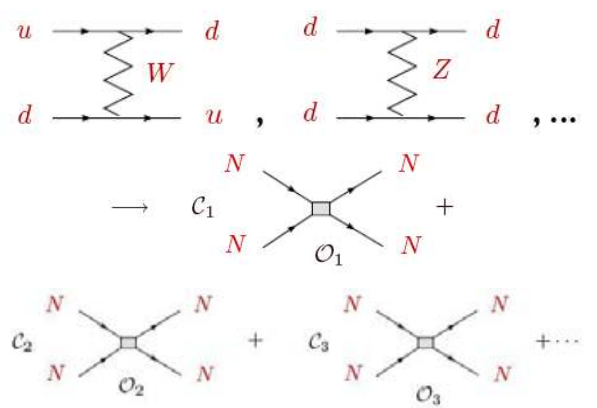

Fig. 3. Quark-gluon-weak-boson PV interactions mapped onto EFT. $N$ is either p or $\mathrm{n}$. The $C$ are unknown parameters. Each box represents an operator.

and in the partial wave basis, we have [19]

$$
\begin{aligned}
& \mathcal{L}_{P V}= \\
& -\left[C^{\left({ }^{3} S_{1}{ }^{1} P_{1}\right)}\left(N^{T} \sigma_{2} \vec{\sigma} \tau_{2} N\right)^{\dagger} \cdot\left(N^{T} \sigma_{2} \tau_{2} i \stackrel{\leftrightarrow}{D} N\right)\right. \\
& +C_{(\Delta I=0)}^{\left({ }^{1} S_{0}{ }^{3} P_{0}\right)} \delta^{a b}\left(N^{T} \sigma_{2} \tau_{2} \tau^{a} N\right)^{\dagger}\left(N^{T} \sigma_{2} \vec{\sigma} \cdot \tau_{2} \tau^{b} i \stackrel{\leftrightarrow}{D} N\right) \\
& +C_{(\Delta I=1)}^{\left({ }^{1} S_{0}{ }^{3} P_{0}\right)} \epsilon^{3 a b}\left(N^{T} \sigma_{2} \tau_{2} \tau^{a} N\right)^{\dagger}\left(N^{T} \sigma_{2} \vec{\sigma} \cdot \tau_{2} \tau^{b} \stackrel{\leftrightarrow}{D} N\right) \\
& +C_{(\Delta I=2)}^{\left({ }^{1} S_{0}{ }^{3} P_{0}\right)} \mathcal{I}^{a b}\left(N^{T} \sigma_{2} \tau_{2} \tau^{a} N\right)^{\dagger}\left(N^{T} \sigma_{2} \vec{\sigma} \cdot \tau_{2} \tau^{b} i \stackrel{\leftrightarrow}{D} N\right) \\
& \left.+C^{\left({ }^{3} S_{1}-{ }^{3} P_{1}\right)} \epsilon^{i j k}\left(N^{T} \sigma_{2} \sigma^{i} \tau_{2} N\right)^{\dagger}\left(N^{T} \sigma_{2} \sigma^{k} \tau_{2} \tau_{3} \stackrel{\leftrightarrow}{D}{ }^{j} N\right)\right] \\
& + \text { h.c., }
\end{aligned}
$$

where $a O \stackrel{\leftrightarrow}{D} b=a O \vec{D} b-(\vec{D} a) O b$ with $O$ some spin-isospinoperator, and

$$
\mathcal{I}=\left(\begin{array}{ccc}
1 & 0 & 0 \\
0 & 1 & 0 \\
0 & 0 & -2
\end{array}\right)
$$

Each $C$ is labeled by the partial waves it connects, and isospin where necessary. Note that the derivatives are gauged to include photon interactions (and these are all of the photon interactions at lowest order). The left-hand parenthetic term in each operator contains the partial wave projection for the $S$-wave, either singlet or triplet as required. The right-hand parenthetic term contains the partial wave projection for the $P$-wave portion of each operator. Note that the placement of the gauged derivative must be kept consistent because it does not commute with the parts of the operator that project onto the appropriate isospin.

Note that these five leading order PV terms were anticipated by Danilov [17]. What is new is the justification of power counting, and the operator and renormalization treatment that becomes the EFT.

\section{Scaling of the weak operators}

The leading order strong interaction terms in the two-nucleon Lagrangian run quickly because of the anomalously large scattering length in the $S$-waves. Because we are in the non-relativistic limit, the four-momentum in Fig. 4 in the loop scales like $Q^{5}$, while each propagator is $1 / Q^{2}$, yielding $\mu \frac{d}{d \mu} C(\mu)=\frac{M \mu}{4 \pi} C^{2}(\mu)$, where $C$ is either the triplet or singlet strong parameter, $\mu$ is the subtraction point, and $M$ is the nucleon mass. The solution is [6,7]

$$
C(\mu)=\frac{4 \pi}{M} \frac{1}{-\mu+1 / a},
$$

where $a$ is the corresponding triplet or singlet scattering length. The $S$-wave "side" of the PV operators is enhanced

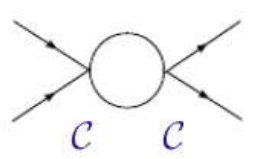

Fig. 4. Mixing of strong $S$ wave operators. $C$ is either the singlet or triplet parameter.

by the same mechanism in Fig. 5, yielding

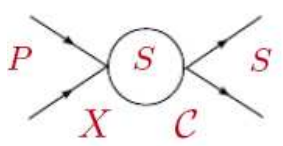

Fig. 5. A PV operator mixing with a strong $S$-wave operator. The PV operator parameter is $X$ and the strong $C$ is either the singlet or triplet, as required. The $P$-wave and $S$-wave portions are labeled.

$$
\mu \frac{d}{d \mu} X(\mu)=\frac{M \mu}{4 \pi} C(\mu) X(\mu),
$$

where $X(\mu)$ is one of the five weak parameters, and $C$ is either the the triplet or singlet strong parameter as needed. The solution is

$$
X(\mu)=X(0) \frac{1 / a}{-\mu+1 / a},
$$

where $a$ is the corresponding triplet or singlet scattering length. As expected, the scaling is the same as in the strong parameter case, and we will see that the PV observables depend upon the scale-independent ratios of the $X$ and the $C$, where $X$ is one of the five PV parameters.

\section{$4 N N$ analyzing power}

The weak portion of the $N N$ asymmetry

$$
A_{L}=\frac{\sigma_{+}-\sigma_{-}}{\sigma_{+}+\sigma_{-}},
$$




\section{p}

Fig. 6. PV $N N$ scattering leading order diagrams. The box is one of the PV operators, the bubble sum in the second term is the $S$-wave rescattering.

is found from Fig. 6. We find [20]

$$
\begin{aligned}
A_{L}^{\vec{n} n} & =32 p \frac{C_{(\Delta I=0)}^{\left({ }^{1} S_{0}{ }^{3} P_{0}\right)}-C_{(\Delta I=1)}^{\left(1 S_{0}-{ }^{3} P_{0}\right)}+C_{(\Delta I=2)}^{\left(1 S_{0}-{ }^{3} P_{0}\right)}}{C_{0}^{\left(1 S_{0}\right)}} \\
A_{L}^{\vec{p} p} & =32 p \frac{C_{(\Delta I=0)}^{\left({ }^{1} S_{0}-{ }^{3} P_{0}\right)}+C_{(\Delta I=1)}^{\left(1 S_{0}-{ }^{3} P_{0}\right)}+C_{(\Delta I=2)}^{\left(1 S_{0}-{ }^{3} P_{0}\right)}}{C_{0}^{\left({ }^{1} S_{0}\right)}} \\
A_{L}^{\vec{n} p} & =32 p \frac{\frac{d \sigma^{1} S_{0}}{d \Omega}}{\frac{d \sigma^{1} S_{0}}{d \Omega}+3 \frac{d \sigma^{3} S_{1}}{d \Omega}} \frac{C_{(\Delta I=0)}^{\left({ }^{1} S_{0}-{ }^{3} P_{0}\right)}-2 C_{(\Delta I=2)}^{\left({ }^{1} S_{0}-{ }^{3} P_{0}\right)}}{C_{0}^{\left(1 S_{0}\right)}} \\
& +32 p \frac{\frac{d \sigma^{3} S_{1}}{d \Omega}}{\frac{d \sigma^{1} S_{0}}{d \Omega}+3 \frac{d \sigma^{3} S_{1}}{d \Omega}} \frac{C^{\left({ }^{3} S_{1}-{ }^{1} P_{1}\right)}-2 C^{\left(3 S_{1}-{ }^{3} P_{1}\right)}}{C_{0}^{\left(3 S_{1}\right)}}
\end{aligned}
$$

where $p$ is the magnitude of the CM momentum and the $\frac{d \sigma}{d \Omega}$ are the leading order parity conserving cross sections. While the $p p$ asymmetry is subject to Coulomb corrections, at these energies and at the angles where experiments are performed the effect is negligible (smaller than the next-to-leading order operators that are neglected here). Note the presence of the $\mu$ independent ratios $X / C_{0}$, as expected.

\section{$5 n p \rightarrow d \gamma$}

The asymmetries involving $n p \rightarrow d \gamma$ come from interference between the strong amplitude in Fig. 7 and the weak amplitude in Fig. 8.

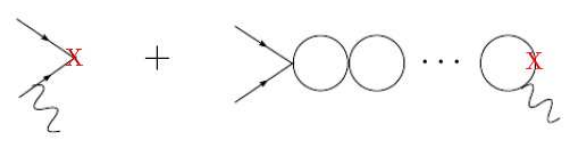

Fig. 7. Diagrams for strong (M1 at this order) $n p \rightarrow d \gamma$. The arrowed lines are nucleons, the wavy lines photons, and the $\mathrm{X}$ stands for the deuteron interpolating field. The bubble sum is from nonperturbative strong scattering.

The amplitude may be written as [21]

$$
\begin{aligned}
\mathcal{M}= & e X N^{T} \tau_{2} \sigma_{2}\left[\boldsymbol{\sigma} \cdot \boldsymbol{q} \boldsymbol{\epsilon}_{d}^{*} \cdot \boldsymbol{\epsilon}_{\gamma}^{*}-\boldsymbol{\sigma} \cdot \boldsymbol{\epsilon}_{\gamma}^{*} \boldsymbol{q} \cdot \boldsymbol{\epsilon}_{d}^{*}\right] N \\
& +i e Y \boldsymbol{\epsilon}^{i j k} \boldsymbol{\epsilon}_{d}^{* i} \boldsymbol{q}^{j} \boldsymbol{\epsilon}_{\gamma}^{* k}\left(N^{T} \tau_{2} \tau_{3} \sigma_{2} N\right) \\
& +i e W \boldsymbol{\epsilon}^{i j k} \boldsymbol{\epsilon}_{d}^{* i} \boldsymbol{\epsilon}_{\gamma}^{* k}\left(N^{T} \tau_{2} \sigma_{2} \sigma^{j} N\right) \\
& +e V \boldsymbol{\epsilon}_{d}^{*} \cdot \boldsymbol{\epsilon}_{\gamma}^{*}\left(N^{T} \tau_{2} \tau_{3} \sigma_{2} N\right)+\ldots
\end{aligned}
$$

where $\boldsymbol{\epsilon}_{\gamma}^{*}$ and $\boldsymbol{\epsilon}_{d}^{*}$ are the polarization vectors for the photon and deuteron, and $\boldsymbol{q}$ is the outgoing photon momentum.
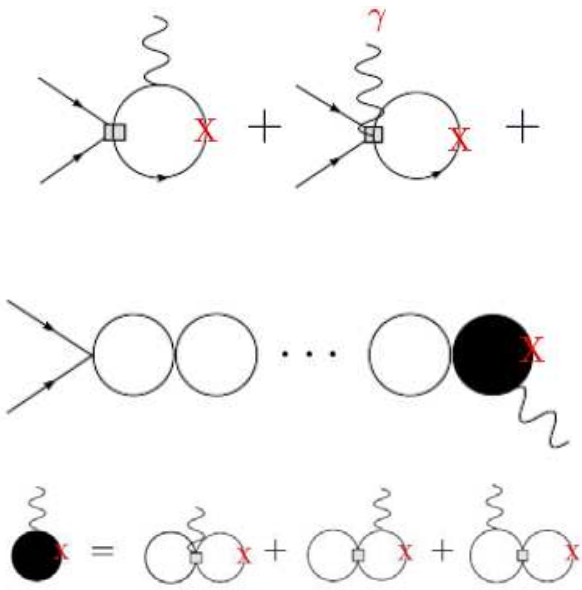

Fig. 8. Diagrams for weak $n p \rightarrow d \gamma$. The box stands for one of the weak operators. The arrowed lines are nucleons, the wavy lines are photons, and the $\mathrm{X}$ is the deuteron interpolating field. The bubble sum is the strong $S$-wave rescattering.

For $\vec{n} p \rightarrow d \gamma$, the PV photon asymmetry is

$$
\begin{gathered}
\frac{1}{\Gamma} \frac{d \Gamma}{d \cos \theta}=1+A_{\gamma} \cos \theta, \\
A_{\gamma}=-2 \frac{M}{\gamma^{2}} \frac{\operatorname{Re}\left[Y^{*} W\right]}{|Y|^{2}},
\end{gathered}
$$

where $\gamma^{2}=M B$, with $B$ the deuteron binding energy. The diagrams in Fig. 7 yield [21] $X=0$ (at this order),

$$
Y=-\frac{2}{M} \sqrt{\frac{\pi}{\gamma^{3}}} \kappa_{1}\left(1-\gamma a^{\left({ }^{1} S_{0}\right)}\right),
$$

and the diagrams in Fig. 8 yield

$$
W=\frac{32 \pi}{3 M} \sqrt{\frac{\gamma}{\pi}} \frac{C^{\left({ }^{3} S_{1}-{ }^{3} P_{1}\right)}}{C_{0}^{\left(3 S_{1}\right)}},
$$

so that

$$
A_{\gamma}=\frac{32}{3} \frac{M}{\kappa_{1}\left(1-\gamma a^{\left(1 S_{0}\right)}\right)} \frac{C^{\left({ }^{3} S_{1}-{ }^{3} P_{1}\right)}}{C_{0}^{\left(3 S_{1}\right)}}
$$

For $n p \rightarrow d \gamma$ the PV circular polarization provides the asymmetry

$$
P_{\gamma}=\frac{\sigma_{+}-\sigma_{-}}{\sigma_{+}+\sigma_{-}}
$$

where $\sigma_{+}\left(\sigma_{-}\right)$is the cross section for photons with positive (negative) helicity.

$$
P_{\gamma}=2 \frac{M}{\gamma^{2}} \frac{\operatorname{Re}\left[Y^{*} V\right]}{|Y|^{2}},
$$

Fig. 8 gives

$$
\begin{aligned}
V= & \frac{16 \pi}{M} \sqrt{\frac{\gamma}{\pi}}\left[\left(1-\frac{2}{3} \gamma a^{\left({ }^{1} S_{0}\right)}\right) \frac{C^{\left({ }^{3} S_{1}-{ }^{1} P_{1}\right)}}{C_{0}^{\left(3 S_{1}\right)}}\right. \\
& \left.+\frac{1}{3} \gamma a^{\left({ }^{1} S_{0}\right)} \frac{C_{(\Delta I=0)}^{\left({ }^{1} S_{0}-{ }^{3} P_{0}\right)}-2 C_{(\Delta I=2)}^{\left({ }^{1} S_{0}-{ }^{3} P_{0}\right)}}{C_{0}^{\left(1 S_{0}\right)}}\right],
\end{aligned}
$$


$19^{\text {th }}$ International IUPAP Conference on Few-Body Problems in Physics

Table 1. The list of two-nucleon parity violating "observables" and the PV $\operatorname{EFT}(\not)$ coefficients from Eqn. 5 they depend upon. $A_{L}^{\vec{n} n}$ and $A_{L}^{\vec{p} p}$ depend upon the same set of parameters, but in different linear combinations. The energy dependence of the $A_{L}^{\vec{n} p},{ }^{1} S_{0}$ and $A_{L}^{\vec{n}, s^{3} S_{1}}$ is different. $A_{\gamma}$ is defined in Eqn. (9). $P_{\gamma}$ is defined in Eqn. (14).

\begin{tabular}{|l|c|c|c|c|c|c|}
\hline Process & $A_{L}^{\overrightarrow{n n} n}$ & $A_{L}^{\vec{p} p}$ & $A_{L}^{\vec{n} p}, S_{0}^{1} S_{0}$ & $A_{L}^{\vec{n} p, S^{3} S_{1}}$ & $A_{\gamma}$ & $P_{\gamma}$ \\
\hline$C^{\left(3 S_{1}-{ }^{1} P_{1}\right)}$ & & & & $\checkmark$ & & $\checkmark$ \\
$C_{(\Delta I=0)}^{\left(1 S_{0}-{ }^{3} P_{0}\right)}$ & $\checkmark$ & $\checkmark$ & $\checkmark$ & & & $\checkmark$ \\
$C_{(\Delta I=1)}^{\left({ }^{(} S_{0}-P_{0}\right)}$ & $\checkmark$ & $\checkmark$ & & & & \\
$\left.C_{(\Delta I=2)}^{\left(1 S_{0}-3\right.} P_{0}\right)$ & $\checkmark$ & $\checkmark$ & $\checkmark$ & & & $\checkmark$ \\
$C^{\left(3 S_{1}-{ }^{3} P_{1}\right)}$ & & & & $\checkmark$ & $\checkmark$ & \\
\hline \hline
\end{tabular}

so that

$$
\begin{array}{r}
P_{\gamma}=-16 \frac{M}{\kappa_{1}\left(1-\gamma a^{\left(1 S_{0}\right)}\right)}\left[\left(1-\frac{2}{3} \gamma a^{\left(1 S_{0}\right)}\right) \frac{C^{\left({ }^{3} S_{1}-{ }^{1} P_{1}\right)}}{C_{0}^{\left(3 S_{1}\right)}}+\right. \\
\left.+\frac{\gamma a^{\left(1 S_{0}\right)}}{3} \frac{C_{(\Delta I=0)}^{\left(1 S_{0}-{ }^{3} P_{0}\right)}-2 C_{(\Delta I=2)}^{\left(1 S_{0}-{ }^{3} P_{0}\right)}}{C_{0}^{\left(1 S_{0}\right)}}\right] .
\end{array}
$$

Once again we notice the ratio of weak-to-strong EFT $(\not)$ parameters in these expressions. Each parameter is individually $\mu$-dependent, but their running is the same, yielding a $\mu$-independent observable as required.

\section{Discussion}

Table 1 collects the two-nucleon observables calculated in $\operatorname{PV} \operatorname{EFT}(\not)$ and the weak unknown parameters upon which they depend. Clearly it will be difficult to measure the analyzing power in $\vec{n} n$ scattering, separate the single from the triplet part of $\vec{n} p$ scattering, etc. In order to extract all of the weak parameters and verify consistency with experiment, more measurements will be needed, likely including three nucleon systems. Fortunately, the same low energy parameters involved in the two nucleon observables will dictate the PV observables in the three body system.

We have shown how to generalize the $\operatorname{EFT}(\not)$ treatment of strong nucleon-nucleon interactions to include parity violation. This is important so that present and future PV experiments can be interpreted in a model-independent systematic and consistent way. The EFT $(\hbar)$ shown should be valid at energies where the pion is not dynamical. This restricts the analysis to energies below $\sim m_{\pi}^{2} / M$. Fortunately the NPDgamma experiment, circular polarized photon break-up, neutron spin rotation, etc. are planned in this energy region.

Clearly more experiments are needed to test this description. But it is a first step towards creating the few body $\operatorname{PV} \operatorname{EFT}(\not)$ that can be used to compare to planned fewbody PV experiments. Only when both are available will it be possible to check the consistency of the description, and determine whether extant measurements are being interpreted correctly.

\section{References}

1. W. Haeberli and B. R. Holstein, arXiv:nuclth/9510062.

2. W. C. Haxton, arXiv:0802.2984 [nucl-th].

3. B. Desplanques, J. F. Donoghue and B. R. Holstein, Annals Phys. 124 (1980) 449.

4. S. L. Zhu, C. M. Maekawa, B. R. Holstein, M. J. Ramsey-Musolf and U. van Kolck, Nucl. Phys. A 748 (2005) 435 [arXiv:nucl-th/0407087].

5. D. B. Kaplan, M. J. Savage and M. B. Wise, Phys. Rev. C 59 (1999) 617 [arXiv:nucl-th/9804032].

6. D. B. Kaplan, M. J. Savage and M. B. Wise, Phys. Lett. B 424 (1998) 390 [arXiv:nucl-th/9801034].

7. D. B. Kaplan, M. J. Savage and M. B. Wise, Nucl. Phys. B 534 (1998) 329 [arXiv:nucl-th/9802075].

8. D. B. Kaplan, M. J. Savage and M. B. Wise, Nucl. Phys. B 478 (1996) 629 [arXiv:nucl-th/9605002].

9. J. W. Chen, G. Rupak and M. J. Savage, Nucl. Phys. A 653 (1999) 386 [arXiv:nucl-th/9902056].

10. G. Rupak, Nucl. Phys. A 678 (2000) 405 [arXiv:nuclth/9911018].

11. P. D. Eversheim et al., Phys. Lett. B 256 (1991) 11; P. D. Eversheim, private communication, 2009; P. D. Eversheim et al., at the Bonn Isochronous Cyclotron," Spring Meeting of the DPG, Salzburg 1992, Abstract in Verhandlungen der DPG (1992) 59; http://parity-experiment.hiskp.uni-bonn.de/

12. V. A. Knyaz'kov et al., Nucl. Phys. A 417 (1984) 209.

13. E. D. Earle et al., Can. J. Phys. 66 (1988) 534.

14. J. F. Cavaignac, B. Vignon and R. Wilson, Phys. Lett. B 67 (1977) 148.

15. J. Alberi et al., Can. J. Phys. 66 (1988) 542.

16. B. Lauss et al., AIP Conf. Proc. 842 (2006) 790 [arXiv:nucl-ex/0601004].

17. G. S. Danilov, Phys. Lett. 18, (1965) 40; B35, (1971) 579; Sov. J . Nucl. Phys. 14, (1972) 443.

18. L. Girlanda, Phys. Rev. C 77 (2008) 067001 [arXiv:0804.0772 [nucl-th]].

19. M. R. Schindler and R. P. Springer, arXiv:0907.5358 [nucl-th].

20. D. R. Phillips, M. R. Schindler and R. P. Springer, Nucl. Phys. A (2009) 8221 [arXiv:0812.2073 [nuclth]].

21. D. B. Kaplan, M. J. Savage, R. P. Springer and M. B. Wise, Phys. Lett. B 449 (1999) 1 [arXiv:nuclth/9807081]. 